\title{
Resistance to various Inhibitors in Aspergillus nidulans
}

\author{
By J. R. WARR* AND J. A. ROPER \\ Department of Genetics, University of Sheffield
}

(Received 2 March 1965)

\begin{abstract}
SUMMARY
Resistant strains of Aspergillus nidulans were obtained by one-step selection on high concentrations of various inhibitors; each strain was mutant in a different gene conferring resistance to actidione $(A c t), p$-fluorophenylalanine $(p f)$, teoquil $(t e)$, iodoacetate $(I o d)$ or malachite green $(m g)$. Some mutant alleles have been firmly, others tentatively, located. For comparative purposes attempts were made to find instances of multi-step or non-genic increases in resistance to malachite green and to teoquil, by prolonged exposure to low concentrations of inhibitor. No such increases were found.

Iod 1 is fully dominant, Act 1 is semidominant, $p f 21$, $t e 1$ and $m g 1$ are recessive. $m g 1$ confers resistance to acriflavine to about the same degree as the non-allelic acr 2 but these two mutant alleles do not show additivity. pf 21 confers resistance to iodoacetate and also suppresses requirement for nicotinic acid (nic 8). Iod 1 strains, which are not resistant to fluoroacetate, are able to use acetate as sole carbon source.

Nutritionally balanced heterokaryons, between Act 1 and sensitive strains, show a gradual (and reversible) increase of the Act component on increasing actidione concentrations. Ultimately a plateau is reached; this presumably represents the nutritional limits of each particular combination of nutritional markers.
\end{abstract}

\section{INTRODUCTION}

Mutant strains of micro-organisms resistant to various agents have been fruitfully applied in diverse genetical and biochemical studies. However, the only detailed studies of resistance in Aspergillus nidulans are those of resistance to acriflavine (Roper \& Käfer, 1957) fluoroacetate (Apirion, 1962) and $p$-fluorophenylalanine (Morpurgo, 1961 b). The present work was undertaken to extend the range of resistant strains of this species and to make initial investigations on their promise as tools in a variety of studies. One-step mutants were deliberately selected as one aspect of the work. However, it would be of interest to compare one-step high resistance with multigenic or non-genic resistance to the same substance. A. nidulans is suitable for the study of extrachromosomal inheritance by the combined use of heterokaryosis, the sexual cycle and parasexual cycle; it is also a potentially favourable organism for the analysis of multigenic inheritance via mitotic analysis. At haploidization there is segregation, without crossing-over, of whole chromosomes; for a first analysis this decreases the number of segregating units to the haploid number of chromosomes. Attempts were made to increase resistance by prolonged exposure to non-inhibitory or slightly inhibitory concentrations of various agents.

* Present address: MRC Biophysics Unit, King's College, 26 Drury Lane, London, W.C. 2. 


\section{METHODS}

The general techniques used throughout this work were those of Pontecorvo Roper, Hemmons, Macdonald \& Bufton (1953). Incubation was at $37^{\circ}$.

Media. Minimal medium (MM), Czapek-Dox with $2 \%$ glucose. Complete medium (CM), a complex medium containing yeast extract, casein hydrolysate, hydrolysed nucleic acid, vitamins, etc. Solid media contained $2 \%$ agar.

Inhibitors. Actidione, iodoacetate, fluoroacetate, $p$-fluorophenylalanine (from Koch-Light \& Co. Ltd, Poyle Trading Estate, Colnbrook, Bucks.), acriflavine (from British Drug Houses, Ltd. Poole, Dorset), malachite green (from G. T. Gurr, Ltd, New King's Road, London, S.W. 6); teoquil, a polymethylene bis isoquinolinium salt with anti-fungal properties (Collier, Potter \& Taylor, 1955) was kindly supplied by Allen \& Hanbury, Ltd. (Ware, Hertfordshire).

Organisms. Strains were taken from laboratory stocks maintained on complete medium CM. Mutant alleles, and their locations, are given by Pontecorvo et al. (1953), Roper \& Käfer (1957), Käfer (1958) and Pontecorvo (1963a). Mutant types used widely in this work were: $y$, yellow conidia, w, white conidia; pyro, bi, paba, ribo, cys, $s, a n, n i c, a d$ and phen designate, respectively, requirement for pyridoxin, biotin, $p$-aminobenzoic acid, riboflavine, cystine, thiosulphate, aneurin (thiamine), nicotinic acid, adenine and phenylalanine; Acr 1 and acr 2, resistance to acriflavine.

Selection of one-step resistant strains. Resistant strains were selected by spreading conidia at high density $\left(10^{6}\right.$ to $\left.2 \times 10^{7} / \mathrm{dish}\right)$ on CM or supplemented MM with a concentration of inhibitor sufficient to suppress completely germination of sensitive conidia. Ultraviolet (u.v.) irradiation (usually to about $2 \%$ survival) was used in most cases. Putative resistant strains were isolated from the highest concentration of inhibitor to give colonies. To avoid clones only one colony was isolated from each plating.

Prolonged exposure to inhibitors. In an attempt to find multi-genic or non-genic adaptation, conidia were spread (20 to 50/dish) on a concentration of inhibitor which permitted full viability but less than full linear growth rate. In one series of experiments a concentration giving only $20 \%$ viability was used. Conidia were taken from a single colony, after 3-4 days of incubation for the next subculture. At intervals survival curves were determined for control and treated organisms.

Survival curves. These provided the most accurate measurement of resistance. They were determined by spreading conidia (20 to 50/dish) on increasing concentrations of inhibitor. About 400 conidia were tested at each concentration of inhibitor. Colony counts were taken at 3 days and expressed as a percentage of a control without inhibitor.

Genetic analysis. Techniques of analysis were, in general, those described by Pontecorvo et al. (1953). Location of a mutant allele to its linkage group via mitotic haploidisation (Forbes, 1959) was facilitated by the $p$-fluorophenylalanine technique (Morpurgo, 1961 $a$; Lhoas, 1961).

\section{RESULTS}

Prolonged exposure to inhibitor

The Aspergillus nidulans strain paba6 $y$; nic 2 was serially subcultured on CM with $0.27 \mathrm{mg}$. malachite green/l. Viability was $100 \%$, but at $70 \mathrm{hr}$ the mean colony 
diameter was $0.6 \mathrm{~cm}$. as compared with $2.6 \mathrm{~cm}$. on inhibitor-free medium. Many hundreds of colonies were examined throughout the experiment but no sectors of vigorous growth were seen. Survival curves were determined for cultures taken after 10, 20 and 30 subcultures; these were compared with a control serially subcultured without inhibitor. No significant difference in resistance was found between the two lines at any time. A further series of seven subcultures were undertaken with the strain $y$; $s$ 12; pyro 4; nic 2 on $\mathrm{CM}$ with $0.4 \mathrm{mg}$. malachite green/l. The viability averaged $20 \%$. Survival curves were determined after each subculture but again no significant change in resistance was found.

Only one series of subcultures was made with teoquil, with strain $y ; s 12 ;$ pyro 4 ; nic 2 grown on $0.5 \mathrm{mg}$. teoquil/l. The viability was $100 \%$. After 15 subcultures no change in resistance was found.

Table 1. Properties of some resistant mutants of Aspergillus nidulans

\begin{tabular}{|c|c|c|c|c|}
\hline \multirow[b]{2}{*}{ Inhibitor } & \multirow{2}{*}{$\begin{array}{l}\text { Mutant } \\
\text { allele* }\end{array}$} & \multirow[b]{2}{*}{ Location } & \multicolumn{2}{|c|}{$\begin{array}{l}\text { Maximum sub- } \\
\text { inhibitory } \\
\text { concentration for } \dagger\end{array}$} \\
\hline & & & Sensitive & Resistant \\
\hline Teoquil & te 1 & $\begin{array}{l}\text { Linkage group III; } 25 \text { units } \\
\text { proximal to } s 12\end{array}$ & $1.5 \mathrm{mg}$. & 3.0 mg. \\
\hline Malachite green & $m g 1$ & $\begin{array}{l}\text { Undetermined. Not in groups I, } \\
\text { II or IV }\end{array}$ & $0.6 \mathrm{mg}$. & $1.0 \mathrm{mg}$. \\
\hline Iodoacetate & $\operatorname{Iod} 1$ & Left arm II; 25 units distal to Act 1 & $1.5 \mathrm{~g}$. & 3.0 g. \\
\hline$p$-Fluorophenylalanine & $p f 21$ & $\begin{array}{l}\text { Left arm } I ; 0.3 \text { units from } \\
\text { ribo } 1\end{array}$ & $0.5 \mathrm{~g}$. & $5 \cdot 0 \mathrm{~g}$. \\
\hline Actidione & Act 1 & $\begin{array}{l}\text { Left arm III. } 8 \text { units from } \\
\text { phen } 4\end{array}$ & $0.7 \mathrm{~g}$. & $2 \cdot 5 \mathrm{~g}$. \\
\hline
\end{tabular}

* Lower case initial for recessive, capital for dominant or semi-dominant. pf alleles numbered from 21 to avoid confusion with mutants of Morpurgo (1961 b).

$\dagger$ Maximum amount/l. complete medium permitting growth by point inoculation of conidia.

\section{One-step mutants}

Five strains of Aspergillus nidulans, each resistant to a different inhibitor, were selected. Details of these strains are shown in Table 1.

Degree of resistance. With the exception of the teoquil resistant strain, all the selected strains could be classified unequivocally by point inoculation of conidia to dishes containing concentrations of inhibitor which completely suppress the growth of sensitive strains. Early tests for sensitivity and resistance on teoquil were often ambiguous. This was due to fragments of mycelium in the inoculum of sensitive strains; such fragments have substantially higher resistance than do conidia; this was shown as follows. Plates of CM without inhibitor were heavily seeded with conidia of a sensitive strain paba $6 y$; nic 2 . An appropriate quantity of teoquil was added at a series of marked places at $1 \mathrm{hr}$ intervals during the first $10 \mathrm{hr}$ of incubation. After 2 days the inhibition zones were measured. There was a decrease in the inhibition zone from the addition at time 0 to that at $5 \mathrm{hr}$, when a plateau was reached. The sharpest decrease in inhibition, between additions at 3 and $5 \mathrm{hr}$, coincided with the germination time. Satisfactory classification of resistance to teoquil could be made only by testing drops of dilute conidial suspensions which were almost free from mycelium. 
Genetic analysis. Meiotic analysis showed that, in each strain, resistance is determined by mutation in a single gene. Both meiotic and mitotic analyses were used to locate the mutant alleles (Table 1). Tests of dominance were made in heterozygous diploids and, in the cases of $\operatorname{Iod} 1$ and $A c t 1$, in heterokaryons balanced with nutritional markers. The complete dominance of $\operatorname{Iod} 1$, and semi-dominance of $A$ ct 1 , held in both heterozygote and heterokaryon.

Resistance to more than one inhibitor. Each resistant strain was tested for increased resistance to all five inhibitors. Acriflavine was also included since it was known (Roper \& Käfer, 1957) that mutations conferring resistance to acriflavine also confer resistance to malachite green. Fluoroacetate was included for comparison with iodoacetate. The only positive results were with $m g 1$ resistant to acriflavine, and pf 21 resistant to iodoacetate and slightly resistant to actidione. Ideally, to eliminate the possible effects of residual genotype, such tests should be carried out on the parent resistant strain and on the resistant recombinants derived from a cross to a sensitive strain. This was done for $m g 1$ but not for $p f 21$.

Malachite green and acriflavine. Because of the cross-resistance effects it was of interest to determine whether acriflavine and malachite green were additive in growth inhibition. To test this, 64 plates of minimal medium were each seeded with $10^{5}$ wild-type conidia. The plates contained various amounts of malachite green and acriflavine. At $65 \mathrm{hr}$ the plates could be sharply classified on the basis of growth or no growth with the result shown in Fig. 1. The two compounds were additive in effect.

The possible interaction of $m g 1$ and $a c r 2$ was also examined. Segregants from the cross $y$; phen $4 \mathrm{mg} 1$; pyro $4 \times a d 15$ paba 1 y; acr 2 were tested by point inoculation on complete medium with acriflavine: $10,15,20,25,30$ and $40 \mathrm{mg}$./l. Of 68 segregants, 49 were resistant, 19 sensitive. This was consistent with a 3:1 ratio and negative interaction of acr 1 and $m g 1$ is excluded. The 49 resistant segregants would have the genotypes acr $2^{+} m g 1$, acr $2 m g 1^{+}$and acr $2 m g 1$. These segregants showed a uniform degree of resistance indistinguishable from that of the parents. Substantial additivity of effect of acr 2 and $m g 1$ was, therefore, excluded.

Interactions of resistance and nutrition. It was shown (McIlwain, 1941; Roper \& Käfer, 1957) that certain nutrients protect against inhibition by acridine; this was found to apply to malachite green. Conidia of sensitive and $m g 1$ strains were suspended in supplemented minimal medium with concentrations of malachite green just sufficient completely to suppress growth. Various additions were made to the plates before incubation. Annullment of inhibition was read as,+ \pm or - , according to growth response around each addition. Responses were: thymus nucleic acid, biotin, riboflavine, $\mathrm{ZnSO}_{4}, \mathrm{Fe}_{3}\left(\mathrm{SO}_{4}\right)_{2}$ and $\mathrm{CuSO}_{4}$, all +; L-cysteine, hydrolysed casein and $\mathrm{MnSO}_{4}$, all \pm . Many other nutrients, including a mixture of 19 amino acids, gave no response. These results applied equally to sensitive and mg 1 strains.

Only annullment by riboflavine was studied further. Conidia of $y$; phen 4 ; pyro 4 or $y$; phen $4 \mathrm{mg} 1$; pyro 4 were spread on dishes of supplemented minimal medium containing various concentrations of malachite green (0-0.6 mg./l.) and riboflavine (0-400 mg./l.). Colony counts were made at 3 days. For both strains riboflavine competitively annulled malachite green inhibition.

A different aspect of the interaction of nutrition and resistance was noted with 
pf 21 . The cross, pf $21 b i 1$; w 3 ; pyro $4 \times$ ribo 1y; Act 1 ; ni 8 , yielded the following segregants with respect to resistance and requirement for nicotinic acid: sensitive, nicotinic acid independent, 88; sensitive, nicotinic acid-requiring, 93; resistantnicotinic acid independent, 171 ; resistant, nicotinic acid-requiring, 0 .

Segregation of resistance versus sensitivity was in the expected 1:1 ratio; among the sensitive segregants, nicotinic acid requirement versus independence segregates as expected. However, there was a total absence of the resistant and nicotinic acid-

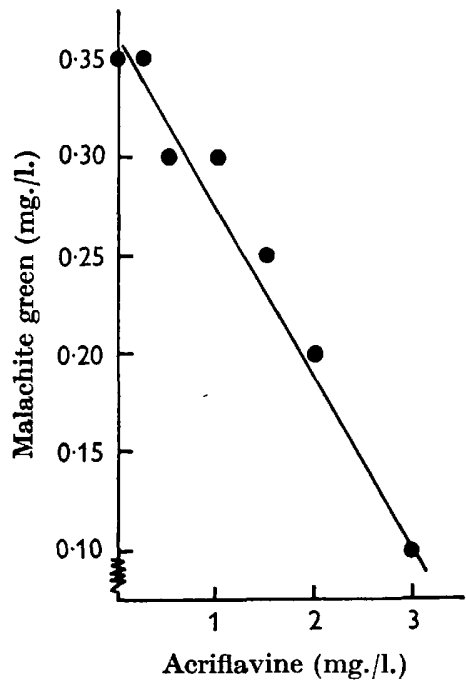

Fig. 1

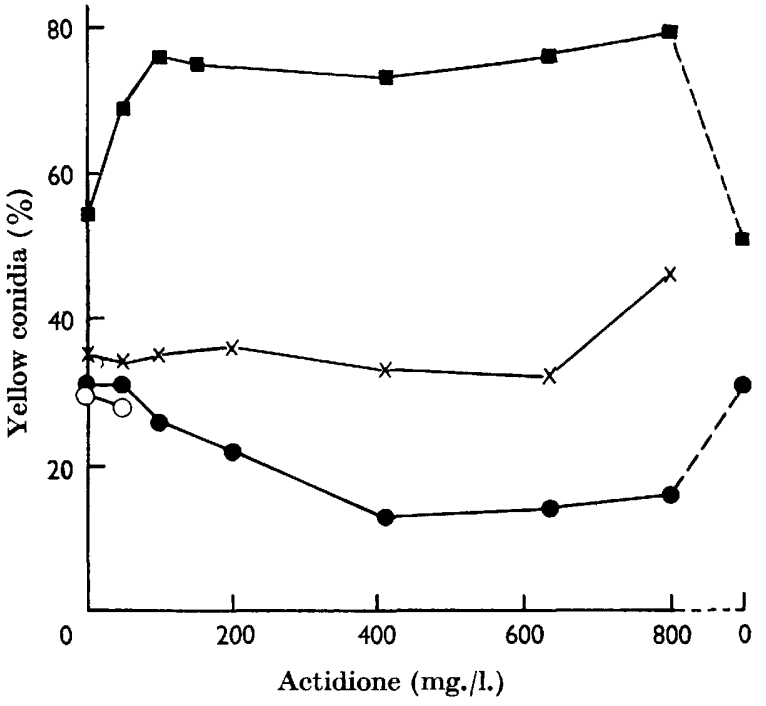

Fig. 2

Fig. 1. Additivity of acriflavine and malachite green in inhibition of growth of wild-type conidia of Aspergillus nidulans. Points represent limits of growth.

Fig. 2. Aspergillus nidulans; adaptive response of heterokaryons. $\bigcirc$, ribo $1 y$; nic 8 with bi 1 ; w 3 ; cys $2 ;$, ribo 1y; Act 1; nic 8 with bi 1 ; w 3 ; cys 2 ;, ribo 1 y; nic 8 with bi 1 ; w 3 ; Act $1 ;$ cys $2 ; \times$, ribo 1y; Act $1 ;$ nic 8 with $b i 1 ;$ w $3 ;$ Act 1 ; cys 2 .

requiring class. The most likely explanation, in view of the otherwise normal phenotypic distributions, was suppression of nic 8 by $p f 21$. Three resistant segregants, potentially carrying nic 8 , were outcrossed to sensitive nicotinic acid independent strains. In all three crosses a 1:1 ratio of sensitive:resistant was found among the segregants. From two crosses there were no segregants which required nicotinic acid. In the third cross, the resistant segregants were all nicotinic acid independent, while half of the sensitives required nicotinic acid.

Expression of Act 1 in heterokaryons. Jinks (1952) was the first to demonstrate adaptive changes in the conidial ratios of heterokaryons in response to changes of media. The heterokaryons were synthesised from wild isolates of Penicillium. Some relatively complex situations have been explored in heterokaryons of Aspergillus nidulans (Pontecorvo et al. 1953; Pontecorvo, $1963 \mathrm{~b}$ ), but heterokaryons balanced on nutritional mutants are generally unsuitable for the exploration of a gradual adaptive change. Act 1, because of its semi-dominance, suggested an alternative approach. 
The following four heterokaryons were established on minimal medium:

1. ribo 1y; nic $8+b i 1 ; w 3$; cys 2 ;

2. $\operatorname{ribo} 1 y ;$ Act $1 ;$ nic $8+b i 1 ;$ w3 3 cys 2 ;

3. ribo 1y; nic $8+b i 1$; w 3 ; Act 1; cys 2 ;

4. ribo 1y; Act 1; nic 8+bi1; w3;Act 1; cys2.

Each heterokaryon was subcultured by transfer of mass hyphal tips, to dishes of minimal medium with various concentrations of actidione. Conidial ratios were determined for these heterokaryons at each actidione concentration. Conidia were randomly sampled from all areas of each colony; the ratio of white:yellow was determined by plating at least $\mathbf{4 0 0}$ conidia from each heterokaryon. The results (Fig. 2) show a clear adaptive response of heterokaryons 2 and 3 . This response is reversed by subculture from actidione back to drug-free minimal medium. Slight differences between heterokaryons 2 and 3 in their degree of response may well be due to differences in residual genotype. The change in heterokaryon 4, at the highest actidione concentration, is probably due to sampling error. The plateau reached by heterokaryons 2 and 3 presumably reflect the nutritional limits of each heterokaryotic combination.

\section{DISCUSSION}

Multi-step or non-genic adaption. Malachite green was chosen for the major part of this work, its choice being dictated by the fact that one-step mutants were available for comparison. Furthermore, Wild \& Hinshelwood (1955) and Bartlett (1959) had shown fungal adaptation to brilliant green whose structure is closely related to that of malachite green. Polygenic resistance is already known (Cavalli \& Maccacaro, 1952; Hotchkiss \& Evans, 1958). Non-genic resistance has been shown for several inhibitors in a variety of organisms (see for example Dean \& Hinshelwood, 1952, 1954); although such adaptation has had a controversial history, it now appears likely that the pnenomenon can be accommodated by ideas such as those of Pollock (1953) and Jacob \& Monod (1961).

In the present studies with malachite green and teoquil, neither multigenic or non-genic increase in resistance emerged at low concentrations of inhibitor. It is impossible to draw any substantial conclusion from such negative results; since the investigations were laborious and unrewarding they were not pursued further. A more profitable approach to multigenic resistance might well be through further selection from the one-step mutants but this has not yet been attempted.

One-step resistance. Resistant strains of Aspergillus nidulans, each mutant in a different gene, were readily obtained for each of the five tested inhibitors. Resistance to so diverse a range of substances shows one facet of the potential biochemical flexibility of the organism. Genetically determined resistance is undoubtedly achieved through any one of a variety of mechanisms; this is to some extent reflected in the different dominance relationships found in mutations to different or even to the same inhibitor (Roper \& Käfer, 1957). No attempt has yet been made to pinpoint the biochemical effects of mutation in the present resistant strains though indirect preliminary evidence is available in some cases. Resistance to iodoacetate is of interest since iodoacetate inhibits a wide range of enzymes. Change in permeability seems a likely mechanism. However, Iod 1 strains did not show increased resistance to fluoroacetate and they were able to use acetate as sole carbon 
source; any change in permeability would need to be very highly selective. Resistance to $p$-fluorophenylalanine might arise through modification of an amino acid activating enzyme (Yanofsky, Helinski \& Maling, 1961; Lewis, 1963) and this might also account for suppression by $p f 21$ of nic 8 . However, it seems reasonable to suppose that a mutation leading to such a change would be semi-dominant rather than recessive. An alternative mechanism of suppressor action, invoking change at the level of the ribosomes, is suggested by the work of Davies, Gilbert \& Gorini (1964). Shikimic acid is a precursor of phenylalanine and nicotinic acid (Davis, $1955)$ and it is possible to suggest yet another mechanism of resistance and nic 8 suppression, based on the increased production of shikimic acid. Morpurgo (1961 b) isolated strains of $A$. nidulans resistant to $p$-fluorophenylalanine and showed that some had a partial requirement for tyyrosine. From a comparison of linkage data (De Palma \& Morpurgo, 1963) it seems very likely that Morpurgo's mutations and ours, which have no requirement for tyrosine, are allelic. If so, the $p f$ locus poses a fascinating problem of pleiotropic effects differently expressed among non-identical allelic mutants. Any explanation of resistance determined by $p f 21$ must also take account of the unexpected cross-resistance to iodoacetate and actidione. Actidione appears to block either transfer of amino acids to ribosomes or polymerisation of amino acids (Siegel \& Sisler, 1963). Both $p f 21$ and Act 1 show actidione resistance but do not yet give any pointers to the mechanisms of resistance. The $A$. nidulans mutant allele $m g 1$, and the mutations studied by Roper \& Käfer (1957), confer resistance to both malachite green and acriflavine. Malachite green and acriflavine are additive in inhibition of wild type and this might indicate a common sensitive site. The non-allelic mutations $m g 1$ and acr 2 are similar in the degree of resistance that each confers but they do not show any interaction in double mutants; this is in contrast to the situation in phage (Hessler, 1963). Annullment of inhibition by acriflavine or malachite green can be achieved with a variety of nutrients and such effects should be interpreted cautiously. Annullment of acriflavine inhibition by nucleic acid (Mcllwain, 1941; Roper \& Käfer, 1957) may be due merely to complexing of the nucleic acid and acriflavine outside the cell. On the other hand, competition between malachite green and riboflavine, both for sensitive and $m g 1$ strains, may reflect relief of inhibition of flavine containing enzymes. Inhibition of such enzymes by acridines is known (Haas, 1944; Hellerman, Lindsay \& Bovarnick, 1946) and alterations in flavine containing enzymes is associated with acridine resistance in pneumococci (Gots \& Sevag, 1948; Sevag \& Gots, 1948a, b). Acridines have now assumed importance as mutagens for chromosomal and non-chromosomal elements and it would be particularly interesting to elucidate the mechanisms of resistance to them.

The main application of the Aspergillus nidulans mutants in the present work has been, for the present, in exploration. All the mutants, except te 1 which is laborious to classify, provide good selective markers for meiotic and mitotic analysis. The present work has shown the ease with which a variety of resistant mutants may be isolated; their differing dominance provides useful flexibility in a variety of genetic studies. Act 1, a semi-dominant allele, has been used to show adaptive changes of conidial ratio in heterokaryons. Such changes could result from differential effects of actidione on the production of conidia by the component strains. But, since a plateau is reached in each adaptive response, it is far more likely that the conidial 
changes reflect changes in nuclear ratio. Balanced heterokaryons of $A$. nidulans generally achieve a particular nuclear ratio dependent on the component strains (Pontecorvo et al. 1953; Clutterbuck, 1964). By use of the present system it should be possible to explore the nutritional limits of particular combinations of nutritional mutants. This could offer an approach, in Aspergillus, to the ideas of Kacser (1963) on rate-limiting reactions.

We are indebted to Dr B. W. Bainbridge and Dr A. J. Clutterbuck for valuable discussion and advice on translocations and heterokaryosis. Messrs Allen \& Hanbury Ltd. kindly supplied teoquil. We also make grateful acknowledgement of a D.S.I.R. Research Studentship to one of us (J.R.W.).

\section{REFERENCES}

ApIrIon, D. (1962). A general system for the automatic selection of auxotrophs from prototrophs and vice versa in micro-organisms. Nature, Lond. 195, 959.

Bartuett, G. W. (1959). Gain and loss of resistance in the fungus Penicillium roqueforti. Proc. roy. Soc. B, 150, 120.

Cavalui, L. L. \& Maccacaro, G. A. (1952). Polygenic inheritance of drug resistance in the bacterium Escherichia coli. Heredity, 6, 311.

Cuutrenbuck, A.J. (1964). Heterokaryosis in Aspergillus nidulans. Ph.D. Thesis. University of Sheffield.

Collier, H. O. J., Potter, M. D. \& TAYlor, E. P. (1955). Antifungal activities of bisisoquinolinium and bisquinolinium salts. Br. J. Pharmacol. 10, 343.

Davis, B. D. (1955). Intermediates in amino acid biosynthesis. Advanc. Enzymol. 16, 247.

Davies, J., Gilbert, W. \& Gorini, L. (1964). Streptomycin, suppression and the code. Proc. nat. Acad. Sci., Wash. 51, 883.

Dean, A. C. R. \& Hinshelwood, C. (1952). The resistance of Bacterium lactis aerogenes to proflavine (2:8diaminoacridine). 1. The applicability of the statistical fluctuation test. Proc. roy. Soc. B, 139, 236.

Dean, A. C. R. \& Hinshelwood, C. (1954). The stability of various adaptations of Bacterium lactis aerogenes (Aerobacter aerogenes). Proc. roy. Soc. B, 142, 45.

de Palma, C. \& Morpurgo, G. (1963). Location of pfp-1. Aspergillus Neres Letter, 4, 11.

Forbes, E. (1959). Use of mitotic segregation for assigning genes to linkage groups in Aspergillus nidulans. Heredity, 13, 67.

Gots, J. S. \& Sevag, M. G. (1948). Enzymatic studies on the mechanism of the resistance of pneumococcus to drugs. I. Studies of the dehydrogenase activities and inter-relationships of pneumococci susceptible and resistant to acriflavine, optochin, propamidine and sulphonamides. J. Bact. 56, 709.

HAAS, E. (1944). The effect of atabrine and quinine on isolated respiratory enzymes. $J$. biol. Chem. 155, 321.

Heliferman, L., Lindsay, A. \& Bovarnick, M. R. (1946). Inhibition of $d$-amino acid oxidase by competition with F.A.D. by atabrine, quinine and certain other drugs. J. biol. Chem. 163, 553.

Hessler, A. Y. (1963). Acridine resistant mutants of $T_{2 H}$ bacteriophage. Genetics, $48,1107$.

Hotchiss, R. D. \& Evans, A. H. (1958). Analysis of the complex sulfonamide resistance locus of Pneumococcus. Cold Spr. Harb. Symp. quant. Biol. 23, 85.

JACOB, F. \& MONOD, J. (1961). Genetic regulatory mechanisms and the synthesis of proteins. J. mol. Biol. 3, 318.

JINKS, J. L. (1952). Heterokaryosis : a system of adaptation in wild fungi. Proc. roy. Soc. B, 140, 83.

KACSER, H. (1963). The kinetic structure of organisms. In Biological Organization. Ed. by R. J. C. Harris, p. 25. New York: Academic Press. 
KäFER, E. (1958). An 8-chromosome map of Aspergillus nidulans. Advanc. Genet. 9, 105.

LEwis, D. (1963). A structural gene for the methionine-activating enzyme and its mutation as a cause of resistance to ethionine. Nature, Lond. 200, 151.

Lноа, P. (1961). Mitotic haploidisation by treatment of Aspergillus niger diploids with $p$-fluorophenylalanine. Nature, Lond. 190, 734.

McIlwain, H. (1941). A nutritional investigation of the antibacterial action of acriflavine. Biochem. J. 35, 1311.

Morpurgo, G. (1961 a). Somatic segregation induced by $p$-fluorophenylalanine. Aspergillus Nerws Letter, $2,4$.

Morpurgo, G. (1961 b). Resistance to antimetabolites in Aspergillus nidulans. Aspergillus Newes Letter, $2,9$.

Pollock, M. R. (1953). Stages in enzyme adaptation. In Adaptation in micro-organisms. Symp. Soc. gen. Microbiol. 3, 150.

Ponteconvo, G. (1963a). Glasgow list of located or partially located mutants of Aspergillus nidulans. Aspergillus News Letter, 4, 12.

Ponteconvo, G. (1963b). Microbial genetics: retrospect and prospect. Proc. roy. Soc. B, $158,1$.

Pontecorvo, G., Roper, J. A., Hemmons, L. M., MacDonald, K. D. \& Bufton, A. W. J. (1953). The genetics of Aspergillus nidulans. Advanc. Genet. 5, 141.

ROPER, J. A. \& Käfer, E. (1957). Acriflavine resistant mutants of Aspergillus nidulans. J. gen. Mierobiol. 16, 660.

SevaG, M. G. \& Gots, J. S. (1948a). Enzymatic studies on the mechanism of the resistance of pneumococcus to drugs. II. The inhibition of dehydrogenase activities by drugs; antagonistic effects of riboflavin to inhibitions. J. Bact. 56, 723.

SevaG, M. G. \& Gots, J. S. (1948b). Enzymatic studies on the mechanism of the resistance of pneumococcus to drugs. III. Experimental results indicating alteration in enzyme proteins associated with the development of resistance to drugs. J. Bact. 56, 737 .

Sieger, M. R. \& Sisler, H. D. (1963). Inhibition of protein synthesis in vitro by cycloheximide. Nature, Lond. 200, 675.

Wild, D. G. \& Hinshelwood, C. (1955). The development of drug resistance in strains of Saccharomyces cerevisiae: resistance to 2:4-dinitrophenol and brilliant green. Proc. roy. Soc. B, 144, 287.

YANOFsky, C., Helsinski, D. R. \& Maling, B. D. (1961). The effects of mutation on the composition and properties of the A protein of Escherichia coli tryptophan synthetase. Cold Spr. Harb. Symp. quant. Biol. 26, 11. 